BARI - TH 169/94, hep-lat/9404017

\title{
Dual Meissner Effect and String Tension in SU(2) Lattice Gauge Theory
}

\author{
Paolo Cea ${ }^{1,2, *}$ and Leonardo Cosmai ${ }^{1, \dagger}$ \\ ${ }^{1}$ Dipartimento di Fisica dell'Università di Bari, 70126 Bari, Italy \\ and \\ ${ }^{2}$ Istituto Nazionale di Fisica Nucleare, Sezione di Bari, 70126 Bari, Italy
}

(April 1994)

\begin{abstract}
We study the distribution of the color fields due to a static quark-antiquark pair in $\mathrm{SU}(2)$ lattice gauge theory. We find that the London penetration length measured after Abelian projection in the Abelian Covariant gauge (Maximal Abelian gauge) agrees with the one obtained without gauge fixing. Moreover the penetration length scales according to asymptotic freedom. We put out a simple relation between the penetration length and the string tension.
\end{abstract}

PACS numbers: 11.15.Ha, 12.38.Aw

Typeset using REVTEX

*Electronic address: cea@bari.infn.it

†Electronic address: cosmai@bari.infn.it 
To understand the non perturbative phenomenon of color confinement in non Abelian gauge theories, G. 't Hooft [1] and S. Mandelstam [2] proposed a model known as dual superconductor model. The physical grounds of the model lie in the theory of superconductivity [3]. In the 't Hooft's formulation the dual superconductor model is elaborated in the framework of the Abelian projection [4]. After a particular gauge has been fixed, reducing the symmetry to that of the maximal Abelian (Cartan) subgroup, the non Abelian gauge theory is described in terms of Abelian projected gauge fields ("photons").

In this scenario there are also color magnetic monopoles whose condensation should cause the confinement of all particles which are color electrically charged with respect to the above photons. Of course this definition of the color magnetic monopoles does depend on the Abelian gauge fixing. However the physics, i.e. the monopole condensation, should be independent on the gauge fixing.

In this paper we analyze the finger-print of the dual superconductor hypothesis, namely the dual Meissner effect. To do this we analyze the distribution of the color fields due to a static quark-antiquark pair in $\mathrm{SU}(2)$ lattice gauge theory. Following Ref. [5] one can explore the field configurations produced by the quark-antiquark pair by measuring the connected correlation function:

$$
\rho_{W}=\frac{\left\langle\operatorname{tr}\left(\mathrm{WLU}_{\mathrm{P}} \mathrm{L}^{\dagger}\right)\right\rangle}{\langle\operatorname{tr}(\mathrm{W})\rangle}-\frac{1}{2} \frac{\left\langle\operatorname{tr}\left(\mathrm{U}_{\mathrm{P}}\right) \operatorname{tr}(\mathrm{W})\right\rangle}{\langle\operatorname{tr}(\mathrm{W})\rangle},
$$

where the plaquette $U_{P}=U_{\mu \nu}(x)$ in the $(\mu, \nu)$ plane is connected to the Wilson loop $W$ through the Schwinger line L. Note that the correlation function (11) is sensitive to the field strength rather than to the square of the field strength $\|$ :

$$
\rho_{W} \underset{a \rightarrow 0}{\longrightarrow} a^{2} g\left[\left\langle F_{\mu \nu}\right\rangle_{q \bar{q}}-\left\langle F_{\mu \nu}\right\rangle_{0}\right] .
$$

According to Eq.(2) we define the color field strength as:

$$
F_{\mu \nu}(x)=\frac{\sqrt{\beta}}{2} \rho_{W}(x) .
$$

By varying the distance and the orientation of the plaquette $U_{P}$ with respect to the Wilson loop $\mathrm{W}$, one can scan the color field distribution of the flux tube. 
We performed numerical simulations on $16^{4}, 20^{4}$, and $24^{4}$ lattices in the range $2.45 \leq$ $\beta \leq 2.7$. We used Wilson loop $L_{W} \times L_{W}, L_{W}=L / 2-2, L$ being the lattice size. In order to eliminate the uninteresting short range quantum fluctuations we cooled our statistical samples. In agreement with previous studies [5,6], the connected correlator $\rho_{W}$ turns out to be sizeable when the plaquette and the Wilson loop are parallel. Thus, the component of the chromoelectric field parallel to the line joining the static charges is sizeable, while the other components of the chromoelectric field and the chromomagnetic field are much smaller. Moreover the longitudinal chromoelectric field is almost constant along the quark-antiquark line, and it decreases rapidly as the transverse distance is increased.

Remarkably enough, in a previous study [7] in the Abelian covariant gauge (maximal Abelian gauge) we found a similar behaviour.

In the continuum the Abelian covariant gauge corresponds to impose the constraints

$$
D_{\mu} A_{\mu}^{ \pm}(x)=0
$$

where $A_{\mu}^{ \pm}=A_{\mu}^{1} \pm i A_{\mu}^{2}$, and $D_{\mu}$ is the $A_{\mu}^{3}$-covariant derivative. On the lattice the constraints (4) are implemented by by maximizing [8]

$$
R=\sum_{x, \mu}\left[\sigma_{3} \widetilde{U}_{\mu}(x) \sigma_{3} \widetilde{U}_{\mu}^{\dagger}(x)\right]
$$

where $\widetilde{U}_{\mu}(x)$ are the gauge-fixed links:

$$
\widetilde{U}_{\mu}(x)=V(x) U_{\mu}(x) V^{\dagger}(x+\hat{\mu})
$$

It is straightforward to check that the residual gauge invariance is the $\mathrm{U}(1)$ group with transformations $g(x)=\exp \left[i \sigma_{3} \theta(x)\right]$.

In the dual superconductor scenario the long range properties of the gauge system are encoded into the Abelian fields. On the lattice the Abelian fields are defined through the Abelian projected links $U_{\mu}^{A}(x)$ [8]:

$$
U_{\mu}^{A}(x)=\operatorname{diag}\left[e^{i \theta_{\mu}^{A}(x)}, e^{-i \theta_{\mu}^{A}(x)}\right], \theta_{\mu}^{A}(x)=\arg \left[\widetilde{U}_{\mu}(x)\right]_{11} .
$$


In Ref. [7] we considered the Abelian projected correlator $\rho_{W}$ :

$$
\rho_{W}^{A}=\frac{\left\langle\operatorname{tr}\left(\mathrm{W}^{\mathrm{A}} \mathrm{U}_{\mathrm{P}}^{\mathrm{A}}\right)\right\rangle}{\left\langle\operatorname{tr}\left(\mathrm{W}^{\mathrm{A}}\right)\right\rangle}-\frac{1}{2} \frac{\left\langle\operatorname{tr}\left(\mathrm{U}_{\mathrm{P}}^{\mathrm{A}}\right) \operatorname{tr}\left(\mathrm{W}^{\mathrm{A}}\right)\right\rangle}{\left\langle\operatorname{tr}\left(\mathrm{W}^{\mathrm{A}}\right)\right\rangle} .
$$

We found that the longitudinal chromoelectric field in the middle of the flux tube can be fitted according to

$$
E_{l}\left(x_{\perp}\right)=\frac{\Phi}{2 \pi} \frac{1}{\lambda^{2}} K_{0}\left(\frac{x_{\perp}}{\lambda}\right), x_{\perp}>0
$$

where $x_{\perp}$ is the transverse distance. $K_{0}$ is the modified Bessel function of order zero, $\Phi$ is the external flux, and $\lambda$ is the London penetration length.

Equation (9) is a straightforward consequence of the dual superconductor hypothesis. Indeed, the transverse shape of the longitudinal chromoelectric field is the dual version of the Abrikosov vortex field distribution. However, the chromoelectric field has been obtained through the gauge-dependent correlator $\rho_{W}^{A}$. So the physical meaning of Eq. (9) is not clear. On the other hand, if the confinement is realized by the dual superconductor mechanism, then Eq.(9) should hold even if we use the gauge invariant correlator $\rho_{W}$. To check this point, we measured $E_{l}\left(x_{\perp}\right)$ in the middle of the flux tube both in the Abelian covariant gauge and without gauge fixing by varying $x_{\perp}$ up to transverse distance $L_{W}$.

Remarkably enough, we find that Eq. (9) holds for both definition of the longitudinal chromoelectric field [9]. We obtain a rather good fit to the data $\left(\chi^{2} /\right.$ d.o.f $\left.\lesssim 1\right)$ if $x_{\perp} \geq 2$ (in lattice units); this is reasonable since Eq. (9) applies in the region $\lambda \gg \xi$, where $\xi$ is the coherence length (more on this later on).

In Figure 1 we report our results for the inverse of the penetration length $\mu=1 / \lambda$. For the gauge invariant correlator $\rho_{W}$ we collected up to 100 configurations with 8 cooling steps (we, however, checked that the results are stable up to 12 cooling steps) at each $\beta$. We performed measurements for 9 different values of $\beta$ in the range $2.45 \leq \beta \leq 2.7$ (for figure readability not all the values are displayed).

In the Abelian covariant gauge we performed 100-500 measurements for 7 different values of $\beta$ in the same range. The gauge is fixed iteratively via the overrelaxation algorithm of Ref. [10] with the overrelaxation parameter $\omega=1.7$. 
Figure 1 suggests that both penetration lengths scale according to two-loop asymptotic scaling law. We find $\mu / \Lambda_{\overline{M S}}=8.9(3)\left(\chi^{2} /\right.$ d.o.f. $\left.\simeq 2\right)$ for the gauge invariant correlator, and $\mu / \Lambda_{\overline{M S}}=8.3(7)\left(\chi^{2} /\right.$ d.o.f. $\left.\simeq 0.4\right)$ in the Abelian covariant gauge. A striking consequence of Fig.1 is that both definitions of the penetration length agree. The overall fit of the data gives (dashed lines)

$$
\frac{\mu}{\Lambda_{\overline{M S}}}=8.8(3)
$$

with $\chi^{2} /$ d.o.f. $\simeq 1$.8. Thus our results strongly suggests that the London penetration length is a physical gauge-invariant quantity.

In Figure 2 we display the external flux $\Phi$ versus $\beta$. Naively we expect that

$$
\Phi=1
$$

Indeed in the Abelian covariant gauge, we see that the data are quite close to Eq. (11). On the other hand, without gauge fixing the external flux is strongly affected by lattice artefacts which seem to disappear by increasing $\beta$. Thus we are led to suspect that the external flux gets renormalized by irrelevant operators, whose effects are strongly reduced in the Abelian covariant gauge. As we will argue in a moment, these effects are responsible for the non-scaling behaviour of the string tension. Indeed, the string tension can be defined as the energy stored into the flux tube per unit length. By observing that the longitudinal chromoelectric field is almost constant along the flux tube, we obtain from Eq. (9):

$$
\sqrt{\sigma}=\frac{\Phi}{\sqrt{8 \pi}} \mu
$$

Now, we have shown that $\mu$ almost scales according to asymptotic freedom. Whence the nonscaling behaviour of the string tension is due to the lattice renormalization of the external charge. However, Eq. (12) allows us to get rid of these effects by putting $\Phi=1$ into Eq. (12):

$$
\sqrt{\sigma}=\frac{\mu}{\sqrt{8 \pi}} .
$$

In Figure 3 we show Eq. (13). For comparison we display also the string tension extracted from the Wilson loops (full points). Due to low statistics and limited lattice size our extimation of the string tension is poor. However, it is gratifying to find that the linear 
extrapolation to the continuum limit of our data is compatible, albeit with large error, with the one obtained on larger lattices [11] (full star):

$$
\frac{\sqrt{\sigma}}{\Lambda_{\overline{M S}}}=1.79 \pm 0.12 \text {. }
$$

The string tension defined by Eq. (13) (open points) seems to scale for $\beta \geq 2.45$ to a value which is consistent with (14). Indeed we find (dashed lines)

$$
\frac{\sqrt{\sigma}}{\Lambda_{\overline{M S}}}=1.76 \pm 0.15
$$

where the quoted error include our extimation of the systematic error.

We would like to comment on the validity of Eq. (13). In order to obtain Eq. (13) we extrapolated Eq. (9) up to $x_{\perp}=0$. This extrapolation leads to a negligible error in (13) if $\lambda / \xi \gtrsim 2$. So it is important to have an extimation of $\xi$. The coherence length is determined by the monopole condensate, the order parameter for the confinement. Unfortunately we do not yet have at disposal a good definition of the order parameter.

Recently two different groups [12,13] give an extimation of the coherence length by using monopole currents defined following DeGrand and Toussaint [14]. However we feel that one can not rely on this approach. As a matter of fact, the authors of Ref. [15] argued convincingly that the DeGrand-Toussaint monopole density is not the order parameter for the confinement.

Let us conclude by stressing the main results of the paper. We found that the London penetration length in the dual Meissner effect is a physical quantity. Moreover we put out a very simple relation between the string tension and the penetration length. A remarkable consequence of our Eq. (9) is that the long range properties the $\mathrm{SU}(2)$ confining vacuum can be described by an effective Abelian theory. In addition, after fixing the gauge with the constraints (4), it seems that the degrees of freedom which are not relevant to the confinement get suppressed. In this respect the situation looks like the Bardeen-CooperSchrieffer (BCS) theory of superconductivity. As it is well known [3], in the BCS theory one considers a reduced Hamiltonian which, however, breaks the electromagnetic gauge 
invariance. Nevertheless, the reduced BCS Hamiltonian, by retaining the degrees of freedom relevant to the superconductivity, gives the correct explanation of the Meissner effect.

Let us conclude by stressing that that the most urgent problem to be addressed in the future studies is the reliable extimation of the coherence length [16]. 


\section{REFERENCES}

[1] G. 't Hooft, in High Energy Physics, Proceedings of the EPS International Conference, Palermo, 1975, Italy, edited by A. Zichichi, (Editrice Compositori, Bologna 1976); G. 't Hooft, Physica Scripta 25, 133 (1982).

[2] S. Mandelstam, Phys. Rep. 23C, 245 (1976).

[3] For a historical overview, see: J. R. Schrieffer, Physics Today, 46, April 1992.

[4] G. 't Hooft, Nucl. Phys. B190 [FS3], 455 (1981).

[5] A. Di Giacomo, M. Maggiore, and Š. Olejnì, Phys. Lett. B236, 199 (1990); Nucl. Phys. B347, 441 (1990).

[6] A. Di Giacomo, Mechanism of Colour Confinement, lecture delivered at the XXXIII Cracow Summer School, Zakopane 1993.

[7] P. Cea and L. Cosmai, Nucl. Phys. B (Proc. Suppl.) 30, 572 (1993).

[8] A. S. Kronfeld, M. L. Laursen, G. Schierholz, and U.-J. Wiese, Phys. Lett. B198, 516 (1987).

[9] Preliminary results on the $16^{4}$ lattice have been presented in the Conference LATTICE '93 : P. Cea and L. Cosmai, preprint BARI-TH 161/93, hep-lat/9311023.

[10] J. E. Mandula and M. Ogilvie, Phys. Lett. B185, 127 (1987).

[11] J. Fingberg, U. Heller, and F. Karsch, Nucl. Phys, B392, 493 (1993).

[12] V. Singh, D. A. Browne, and R. Haymaker, Nucl Phys B (Proc. Suppl.) 30, 568 (1993); Phys. Lett. B306, 115 (1993).

[13] Y. Matsubara, S. Ejiri, and T. Suzuki, talk given in the LATTICE '93 Conference, hep-lat/9311061.

[14] T. A. DeGrand and D. Toussaint, Phys. Rev. D22, 2478 (1980). 
[15] L. Del Debbio, A. Di Giacomo, M. Maggiore, and Š. Olejnik, Phys. Lett. B267, 254 (1991).

[16] A promising proposal is discussed in: L. Del Debbio, A. Di Giacomo, and G. Paffuti IFUP-TH 16/94, hep-lat/9403013. 


\section{FIGURES}

FIG. 1. The inverse of the penetration length in units of $\Lambda_{\overline{M S}}$ as a function of $a \Lambda_{\overline{M S}}$. Unfixed gauge: circles $L=16$, triangles $L=20$, square $L=24$. Abelian covariant gauge: crosses $L=16$, diamond $L=20$.

FIG. 2. The external flux versus $\beta$. Symbols as in Fig.1.

FIG. 3. The square root of the string tension in units of $\Lambda_{\overline{M S}}$ versus $a \Lambda_{\overline{M S}}$. Open symbols refer to Eq. (13). Full star is the extrapolated continuum limit. 
This figure "fig1-1.png" is available in "png" format from: http://arxiv.org/ps/hep-lat/9404017v1 
This figure "fig1-2.png" is available in "png" format from: http://arxiv.org/ps/hep-lat/9404017v1 
This figure "fig1-3.png" is available in "png" format from: http://arxiv.org/ps/hep-lat/9404017v1 